\title{
A Proposal for Modification of the Barcelona Clinic Liver Cancer Staging System Considering the Prognostic Implication of Performance Status
}

Hyo Jung Cho ${ }^{1}$, Soon Sun Kim ${ }^{1}$, So Young Kang ${ }^{2}$, Min Jae Yang ${ }^{1}$, Choong Kyun Noh ${ }^{1}$, Jae Chul Hwang ${ }^{1}$, Sun Gyo Lim ${ }^{1}$, Sung Jae Shin ${ }^{1}$, Kee Myung Lee ${ }^{1}$, Byung Moo Yoo ${ }^{1}$, Kwang Jae Lee ${ }^{1}$, Jin Hong Kim ${ }^{1}$, Sung Won Cho ${ }^{1}$, and Jae Youn Cheong ${ }^{1}$, on behalf of the Korean Liver Cancer Association

${ }^{\prime}$ Department of Gastroenterology, and ${ }^{2}$ Office of Biostatistics, Ajou University School of Medicine, Suwon, Korea

Background/Aims: Barcelona Clinic Liver Cancer (BCLC) C stage demonstrates considerable heterogeneity because it includes patients with either symptomatic tumors (performance status [PS], 1-2) or with an invasive tumoral pattern reflected by the presence of vascular invasion (VI) or extrahepatic spread (EHS). This study aimed to derive a more relevant staging system by modification of the BCLC system considering the prognostic implication of PS. Methods: A total of 7,501 subjects who were registered in the Korean multicenter hepatocellular carcinoma (HCC) registry database from 2008 to 2013 were analyzed. The relative goodness-of-fit between staging systems was compared using the Akaike information criterion (AIC) and integrated area under the curve (IAUC). Three modified BCLC (m-BCLC) systems (\#1, \#2, and \#3) were devised by reducing the role of PS. Results: As a result, the BCLC C stage, which includes patients with PS 1-2 without VI/EHS, was reassigned to stage 0 , A, or B according to their tumor burden in the m-BCLC \#2 model. This model was identified as the most explanatory and desirable model for HCC staging by demonstrating the smallest $\mathrm{AIC}(\mathrm{AIC}=70,088.01)$ and the largest IAUC (IAUC=0.722), while the original BCLC showed the largest AIC (AIC=70,697.17) and the smallest IAUC (IAUC=0.705). The m-BCLC \#2 stage $C$ was further subclassified into $C 1, C 2$, $\mathrm{C} 3$, and $\mathrm{C} 4$ according to the Child-Pugh score, PS, presence of EHS, and tumor extent. The C1 to C4 subgroups showed significantly different overall survival distribution between groups ( $p<0.001)$. Conclusions: An accurate and relevant staging system for patients with HCC was derived though modification of the BCLC system based on PS. (Gut Liver 2019;13:557-568 )
Key Words: Hepatocellular carcinoma; Stage; Barcelona Clinic Liver Cancer Staging; Eastern Cooperative Oncology Group performance status

\section{INTRODUCTION}

The Barcelona Clinic Liver Cancer (BCLC) staging system is the most frequently used staging system among patients with hepatocellular carcinoma (HCC) worldwide. ${ }^{1,2}$ Major leading international liver study groups such as the American Association for the Study of Liver Diseases, the American Gastroenterological Association, and the European Association for the Study of Liver have endorsed the BCLC staging system. ${ }^{1,3}$ However, the BCLC staging system has several limitations. ${ }^{4}$ One of the most well-known limitations of the BCLC is the heterogeneity of the BCLC B. ${ }^{5,6}$ The heterogeneity of BCLC B has been the center of controversy in recent years, and numerous studies for appropriate subclassification of BCLC B stage have been reported., ${ }^{5,7}$ Compared with BCLC B stage, the heterogeneity of BCLC C stage has received relatively little attention. However, it is also an important problem that hinders the clinical application of the BCLC system. According to the definition of the BCLC system, C stage comprises patients with either symptomatic HCC (performance status [PS], 1-2), or with an invasive tumoral pattern reflected by the presence of vascular invasion (VI) or extrahepatic spread (EHS). ${ }^{1,9}$ According to this definition, the patients with PS 1-2 but without EHS/VI are also categorized to C stage, and it causes considerable heterogeneity in the aspect of tumor burden.

PS is generally considered as a good physical parameter and prognostic indicator in cancer patients. ${ }^{10,11}$ Nevertheless, PS has several considerable limitations in patients with HCC. First, PS is

Correspondence to: Jae Youn Cheong

Department of Gastroenterology, Ajou University School of Medicine, 164 World cup-ro, Yeongtong-gu, Suwon 16499, Korea

Tel: +82-31-219-6939, Fax: +82-31-219-5999, E-mail: jaeyoun620@gmail.com

Received on October 2, 2018. Revised on November 19, 2018. Accepted on December 13, 2018. Published online May 30,2019 pISSN 1976-2283 eISSN 2005-1212 https://doi.org/10.5009/gnl18444

() This is an Open Access article distributed under the terms of the Creative Commons Attribution Non-Commercial License (http://creativecommons.org/licenses/by-nc/4.0) which permits unrestricted non-commercial use, distribution, and reproduction in any medium, provided the original work is properly cited. 
subjective, difficult to define, and an immeasurable parameter. Thus, PS might be reported inaccurately and its reproducibility often does not meet expectation. ${ }^{12,13}$ And, it is hard to discriminate whether the symptoms are caused by tumor itself or by the other issues including underlying liver cirrhosis. Despite the aforementioned limitations, PS is one of the major determinants for stratifying HCC stage in the BCLC system, particularly for BCLC stage C. Therefore, verifying whether it is better to maintain the present BCLC system despite the heterogeneity caused by PS or to modify the BCLC system by reducing the role of PS and allocating weight to the tumor extent is necessary.

In this study, we aimed to assess the prognostic implication of PS in the BCLC staging system and to propose modification of the BCLC staging system by considering the prognostic impact of PS in patients with HCC. We also proposed the subclassification of BCLC stage $\mathrm{C}$ to facilitate treatment decision in clinical practice.

\section{MATERIALS AND METHODS}

\section{Data extraction and definitions}

The nationwide Korean multicenter HCC registry database provided by the Korean Liver Cancer Study Group (KLCSG) was analyzed. The KLCSG cohort was constructed by random sampling of the Korean Central Cancer Registry database. It was composed of three databases set according to first patient visit date with HCC diagnosis. Among the 2003 to $2005(n=4,520)$, 2008 to $2010(n=4,596)$, and 2011 to $2012(n=3,119)$ databases, only the latter two databases were analyzed because BCLC staging and PS were not available in 2003 to 2005 database.

The database of KLCSG cohort contained clinical information regarding age, sex, date of diagnosis, cause of underlying liver disease, Child-Pugh (CP) classification, tumor number and size, presence of VI/nodal spread/EHS, serum albumin, bilirubin, international normalized ratio, platelet count, modified Union for International Cancer Control (UICC) stage, BCLC stage, Eastern Cooperative Oncology Group (ECOG) PS scale, ${ }^{10}$ initial treatment modality, initial treatment date, and overall survival (OS).

The diagnosis of HCC was made by the results of typical radiological features, such as early arterial enhancement and delayed wash-out in at least two dynamic imaging modalities including multiphasic spiral computed tomography, dynamic magnetic resonance imaging, and arteriography, or by one single positive imaging technique with serum $\alpha$-fetoprotein levels $\geq 400 \mathrm{ng} / \mathrm{mL}^{1}$

The up-to-7 (UT7) criterion was defined as the sum of the tumor number and the diameter of the largest tumor. ${ }^{14}$ To meet the UT7 criterion, the sum of scores should not be more than 7 . If the sum of the tumor number and the largest diameter exceed 7, the subjects could not meet the UT7 criterion. The design and procedure of the present study was approved by the Institutional Review Board of the Ajou University Hospital, Suwon, South
Table 1. Baseline Characteristics of Included Patients

\begin{tabular}{|c|c|c|}
\hline Characteristic & $\begin{array}{c}\text { All patients } \\
(\mathrm{n}=7,501)\end{array}$ & $\begin{array}{l}\text { BCLC stage C patients } \\
\qquad(\mathrm{n}=2,534)\end{array}$ \\
\hline Age, yr & $59.6 \pm 11.7$ & $58.5 \pm 12.1$ \\
\hline Male sex & $5,930(79.1)$ & $2,078(82.0)$ \\
\hline \multicolumn{3}{|l|}{ Etiology } \\
\hline $\mathrm{HBV}$ & 4,547 (60.6) & $1,573(62.1)$ \\
\hline $\mathrm{HCV}$ & 798 (10.6) & $208(8.2)$ \\
\hline $\mathrm{HBV} \& \mathrm{HCV}$ & $103(1.4)$ & $44(1.7)$ \\
\hline Alcohol & $905(12.1)$ & 299 (11.8) \\
\hline Others & $1,148(15.3)$ & $410(16.2)$ \\
\hline \multicolumn{3}{|l|}{ Child-Pugh class } \\
\hline A & 5,295 (70.6) & $1,549(61.1)$ \\
\hline B & $1,748(23.3)$ & 964 (38.0) \\
\hline $\mathrm{C}$ & $370(4.9)$ & 0 \\
\hline Tumor size, cm & $5.1 \pm 3.73$ & $7.43 \pm 3.70$ \\
\hline \multicolumn{3}{|l|}{ Tumor number } \\
\hline 1 & 4,571 (60.9) & $1,244(49.1)$ \\
\hline 2 & 1,047 (14.0) & $270(10.7)$ \\
\hline 3 & 296 (3.9) & $89(3.5)$ \\
\hline 4 & $113(1.5)$ & $36(1.4)$ \\
\hline$\geq 5$ & $1,165(15.5)$ & 888 (35.0) \\
\hline \multicolumn{3}{|l|}{ Performance status } \\
\hline 0 & $4,128(55.0)$ & 928 (36.6) \\
\hline 1 & $844(11.3)$ & 755 (29.8) \\
\hline 2 & $211(2.8)$ & $169(6.7)$ \\
\hline 3 & $94(1.3)$ & 0 \\
\hline 4 & $68(0.9)$ & 0 \\
\hline BCLC stage & & - \\
\hline 0 & 665 (8.9) & \\
\hline A & $2,952(39.4)$ & \\
\hline B & 853 (11.4) & \\
\hline $\mathrm{C}$ & 2,534 (33.8) & \\
\hline $\mathrm{D}$ & 497 (6.6) & \\
\hline \multicolumn{3}{|l|}{ Modified UICC stage } \\
\hline I & 1,109 (14.8) & $72(2.8)$ \\
\hline II & 2,827 (37.7) & $262(10.3)$ \\
\hline III & $1,994(26.6)$ & $737(29.1)$ \\
\hline IVa & $935(12.5)$ & 785 (31.0) \\
\hline $\mathrm{IVb}$ & 775 (10.3) & $651(25.7)$ \\
\hline First treatment option & - & \\
\hline Resection & & $203(8.0)$ \\
\hline Liver transplantation & & $10(0.4)$ \\
\hline Local ablation therapy & & $72(2.8)$ \\
\hline Transarterial therapy & & $1,107(43.7)$ \\
\hline Sorafenib & & $167(6.6)$ \\
\hline $\begin{array}{l}\text { Systemic therapy other } \\
\text { than sorafenib }\end{array}$ & & $133(5.3)$ \\
\hline Radiation therapy & & $76(3.0)$ \\
\hline Conservative care & & 691 (27.3) \\
\hline
\end{tabular}

Data are presented as mean \pm SD or number (\%).

BCLC, Barcelona Clinic Liver Cancer; HBV, hepatitis B virus; HCV, hepatitis C virus; UICC, Union for International Cancer Control. 
Korea (IRB number: AJIRB-MED-MDB-18-017). The informed consent was waived.

\section{Statistical analysis}

Statistical analysis was performed using SPSS software version 19.0 (IBM Corp., Armonk, NY, USA) and R software version 3.2.5 (R Development Core Team, R: A language and environment for statistical computing; R Foundation for Statistical Computing, Vienna, Austria http://www.R-project.org).

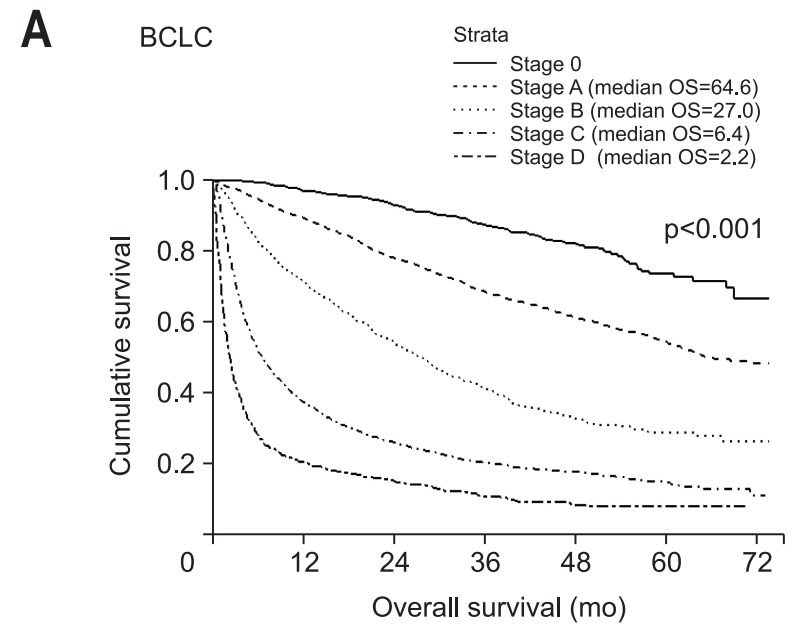

No. of patients at risk

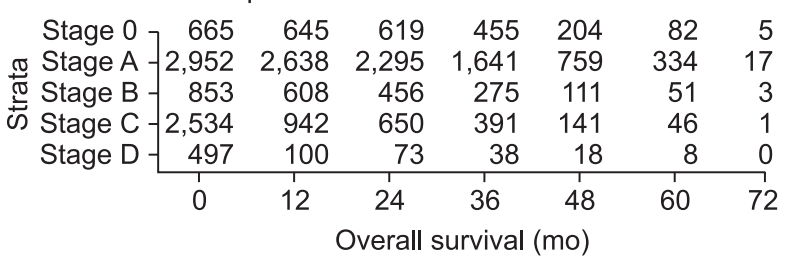

\section{C}

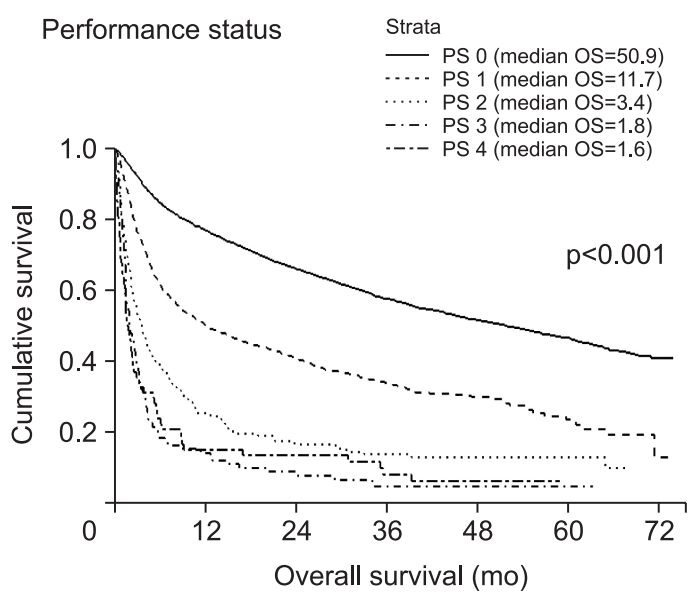

No. of patients at risk

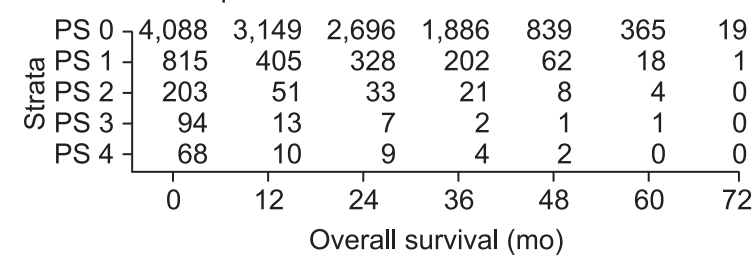

To compare OS of the patients according to BCLC stage or PS, we performed the Kaplan-Meier survival analysis with the logrank test. The Akaike information criterion (AIC) was used as a measure of relative goodness-of-fit to compare the prognostic accuracy of the original BCLC and modified BCLC model. A smaller AIC value suggests a more explanatory and desirable model to predict prognosis. To compare prognostic capacity, the values of integrated area under the curves (IAUC) of each model were calculated and compared. To identify prognostic factors

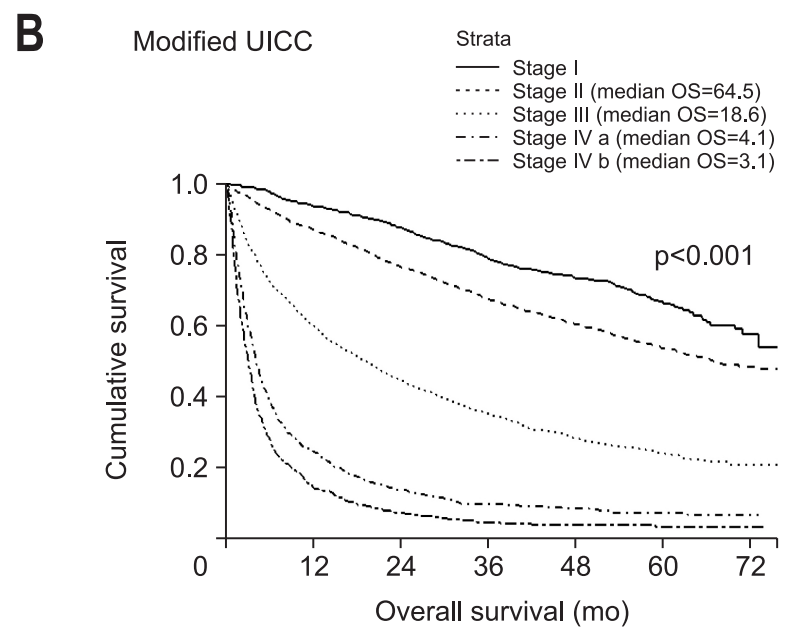

No. of patients at risk

\begin{tabular}{|c|c|c|c|c|c|c|c|}
\hline Stage I & 1,093 & 1,023 & 951 & 666 & 306 & 132 & 7 \\
\hline ఠ్ Stage II & 2,749 & 2,393 & 2,090 & 1,495 & 666 & 285 & 14 \\
\hline సٓ Stage III & $-1,952$ & 1,162 & 852 & 525 & 218 & 86 & 5 \\
\hline ڤ Stage IV a & 912 & 222 & 124 & 68 & 25 & 11 & 0 \\
\hline \multirow{3}{*}{ Stage IV b } & 750 & 105 & 53 & 28 & 10 & 5 & 0 \\
\hline & 0 & 12 & 24 & 36 & 48 & 60 & 72 \\
\hline & \multicolumn{6}{|c|}{ Overall survival (mo) } & \\
\hline
\end{tabular}

Fig. 1. Comparison of overall survival (OS) according to Barcelona Clinic Liver Cancer (BCLC) stage, modified Union for International Cancer Control (UICC), and performance status (PS) in all patients. (A) Comparison of OS according to BCLC. (B) Comparison of OS according to modified UICC. (C) Comparison of OS according to PS in all patients. 
of OS, univariate and multivariate Cox regression analyses were performed. Hazard ratios (HRs) were calculated with 95\% confidence intervals (CIs). A p-value of $<0.05$ (two-sided) was considered statistically significant.

\section{Baseline characteristics of enrolled patients}

Among the 7,715 patients, 214 without BCLC stage information were excluded and data of 7,501 patients were analyzed. The median follow-up period of the enrolled patients was 27.1 months (range, 0 to 73 months). Table 1 shows baseline characteristics of the included patients. The patients consisted of 5,930 men (79.1\%) and 1,571 women (20.9\%) aged 59.63 \pm 11.65 years. The main cause of underlying liver disease was hepatitis B virus (HBV) in 4,547 patients (60.6\%). Hepatitis C virus (HCV), alcohol, HBV/HCV coinfection, and other causes were accounted for 798 (10.6\%), 90 5(12.1\%), 103 (1.4\%), and 1,148 (15.3\%) patients, respectively. In CP classification, 5,295 patients (70.6\%) were classified as having CP class A, and 1,748 (23.3\%) and 370 (4.9\%) patients were classified as having CP classes B and C, respectively.

In the aspect of BCLC stage, 665 (8.95\%), 2,952 (39.4\%), 853 (11.4\%), 2,534 (33.8\%), and 497 (6.6\%) patients had cancer stages A, B, C and D, respectively. When we classified the patients according to the modified UICC stage, 1,093 (14.6\%), 2,749 (36.6\%), 1,952 (26.0\%), 912 (12.2\%), and 750 (10.0\%) patients were categorized into modified UICC stages I, II, III, IVa, and $\mathrm{IVb}$, respectively. In terms of PS, 4,088 patients (54.5\%) were categorized into PS 0, and 815 (10.9\%), 203 (2.7\%), 94 (1.3\%), and $68(0.9 \%)$ patients were categorized into PS 1, 2, 3, and 4, respectively. Fig. 1 shows the comparison of OS according to BCLC stage, modified UICC, and PS. The OS distributions were

A BCLC stage C

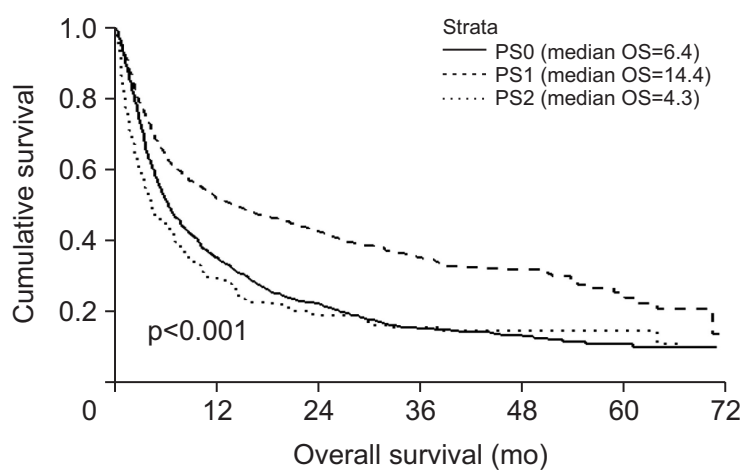

No. of patients at risk

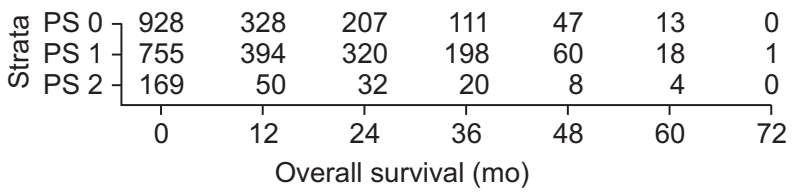

significantly different according to BCLC stage, modified UICC stage, and PS. The patients with more advanced stage showed significantly poor OS both in BCLC stage and modified UICC stage. The distribution of OS was significantly different across PS 0 to 3; however, no significant difference was found between PS 3 and 4 ( $\mathrm{p}=0.836)$.

\section{RESULTS}

\section{Heterogeneity of BCLC C}

Baseline characteristics of the patients with BCLC C stage are reported in Table 1 . The heterogeneity of the BCLC $\mathrm{C}$ stage was reflected by several variables. According to the modified UICC stage, which was derived from the TNM stage by considering only the tumor factors, the patients with BCLC C stage were redistributed according to modified UICC I (72 patients, 2.8\%), II (262 patients, 10.3\%), III (737 patients, 29.1\%), IVa (785 patients, 31.0\%), and IVb (651 patients, 25.7\%). This result represents the heterogeneity of the BCLC C stage in the aspect of tumor extent. The heterogeneity of the BCLC C stage mainly resulted from PS 1-2 patients without any VI or EHS being categorized under BCLC C considering PS. It also caused an unexpected result in the survival analysis according to PS in C stage (Fig. 2). Generally, better PS implicates better OS; however, the patients with PS 1 had significantly better OS than those with PS 0 in BCLC $\mathrm{C}$ stage. The heterogeneity of the BCLC C stage also caused discrepancies between treatment guidelines proposed by the BCLC system and real clinical practice. The patients with BCLC C stage were treated with various treatment strategies including surgical resection, radiofrequency ablation, transarterial therapy, or sorafenib.

B Modified BCLC \#2 model C

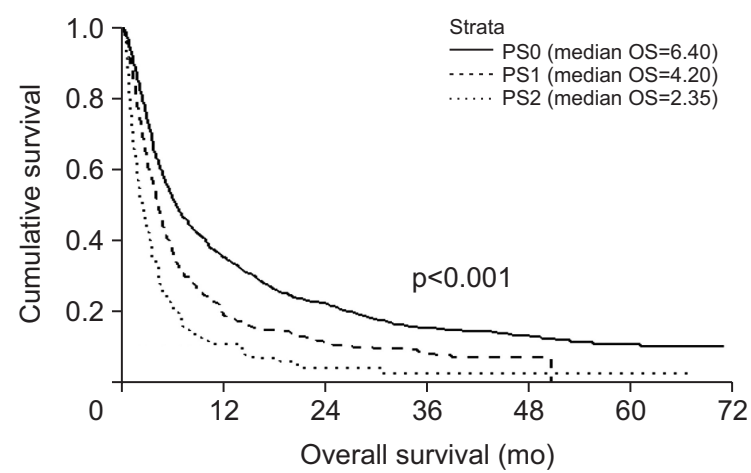

No. of patients at risk

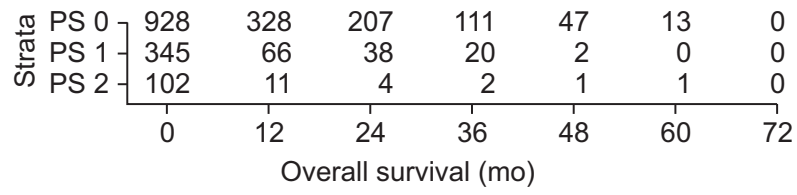

Fig. 2. Comparison of overall survival (OS) according to performance status (PS). (A) Comparison of OS according to performance status (PS) in original Barcelona Clinic Liver Cancer (BCLC) stage C. (B) Comparison of OS according to PS in the modified BCLC \#2 model stage C. 


\section{Suggestion of modification of the BCLC system}

As demonstrated in the above section, BCLC C stage represents considerable heterogeneity in the aspect of tumor extent. It causes discrepancies between treatment guidelines and real clinical practice. Therefore, we devised modified BCLC models by reducing the role of PS and giving more weight to tumor extent.

Two modified BCLC systems (m-BCLC \#1, \#2) were derived by re-allocating the patients with PS 1-2 and without any VI/ EHS (Fig. 3). At m-BCLC \#1, patients with PS 1 (no VI, no EHS) were re-allocated into BCLC 0 , A, or B according to their tumor burden, whereas both of patients with PS 1 and 2 were re-allocated at m-BCLC \#2. While m-BCLC \#1 and \#2 were devised by discussion of four hepatologists in Ajou University Hospital (H.J.C., J.Y.C., S.W.C., and S.S.K), m-BCLC \#3 was the modified model derived by Hsu et al.$^{15}$ Hsu et al. proposed three modified BCLC models (\#A, \#B, and \#C) based on PS. They suggested that \#C model (the same model with m-BCLC \#3 in our study) is the most explanatory model after comparison of prognostic implication between the modified models. m-BCLC \#3 led to re-allocation of patients with PS 1 (no VI, no EHS) into BCLC B stage, although they were originally classified into BCLC C stage. Fig. 4 shows the comparison of OS distribution according to the stage in the m-BCLC models. All of the staging systems including the original BCLC system and m-BCLCs showed significantly different $0 S$ distribution from stage 0 to $\mathrm{D}$ (all $\mathrm{p}<0.001$ ). Table 2 shows the results of Cox regression analy-

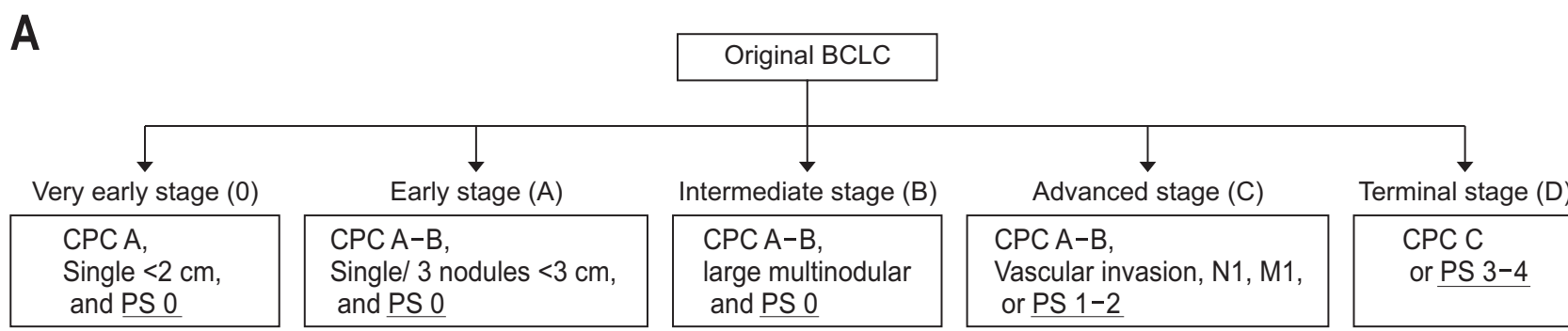

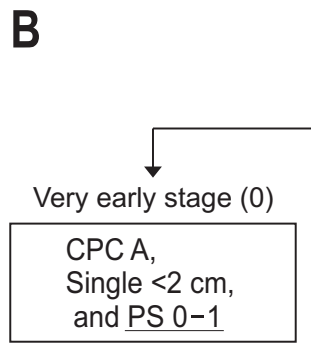

C

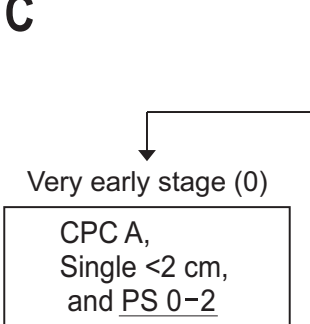

D

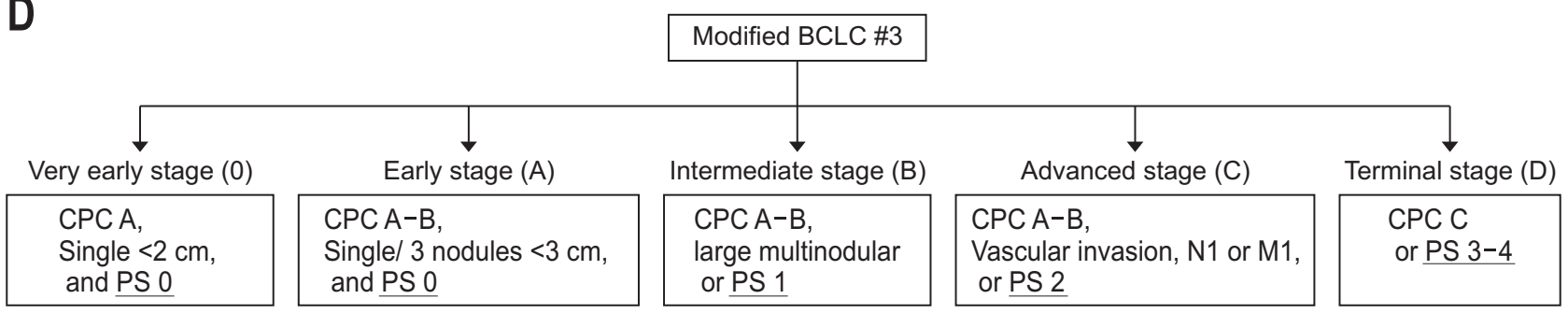

Fig. 3. Proposal for modification of Barcelona Clinic Liver Cancer (BCLC) system. (A) Original BCLC system. (B) Modified BCLC \# 1 model. (C) Modified BCLC \#2 model. (D) Modified BCLC \#3 model.

CPC, Child-Pugh class; PS, performance status. 
A

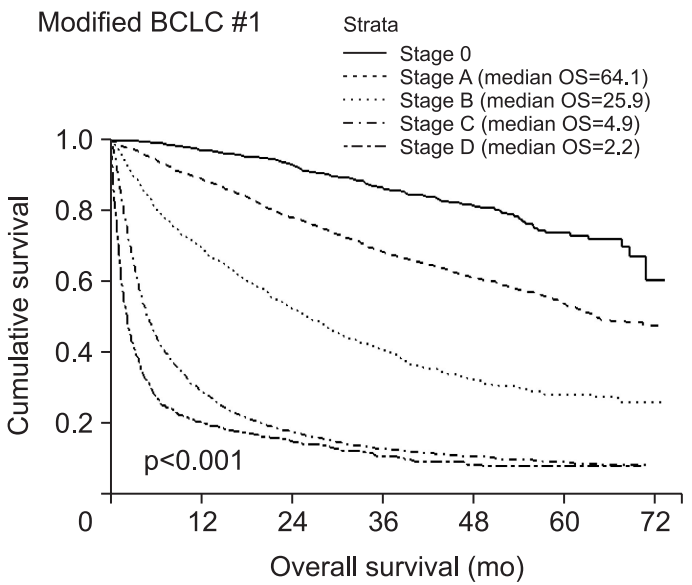

No. of patients at risk

\begin{tabular}{|c|c|c|c|c|c|c|c|}
\hline Stage 0 & 732 & 712 & 682 & 495 & 218 & 88 & 5 \\
\hline Stage A & 3,110 & 2,778 & 2,425 & 1,728 & 789 & 343 & 18 \\
\hline Stage B & 1,048 & 731 & 547 & 328 & 126 & 54 & 3 \\
\hline Stage C & 2,114 & 612 & 366 & 211 & 82 & 28 & 0 \\
\hline \multirow{3}{*}{ Stage D } & 497 & 100 & 73 & 38 & 18 & 8 & 0 \\
\hline & 0 & 12 & 24 & 36 & 48 & 60 & 72 \\
\hline & \multicolumn{7}{|c|}{ Overall survival (mo) } \\
\hline
\end{tabular}

C

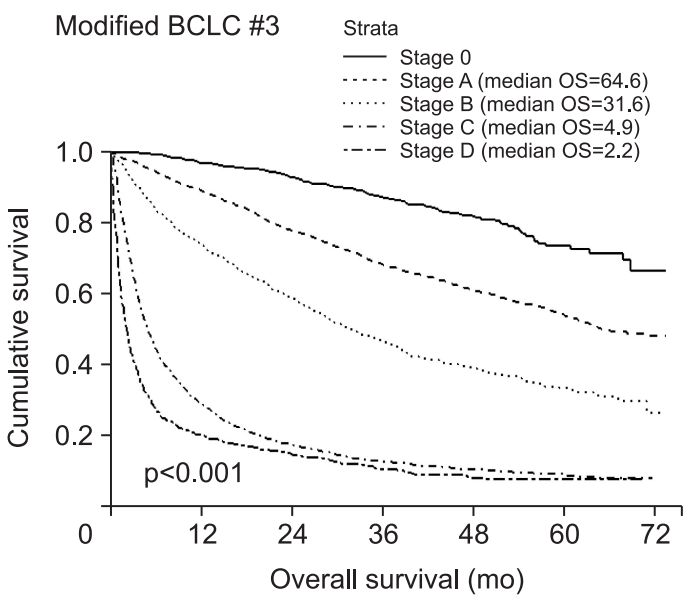

No. of patients at risk

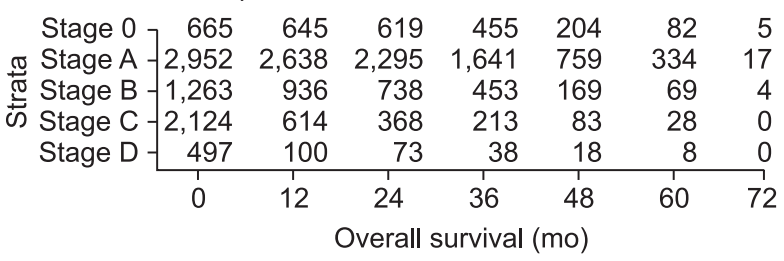

sis and AIC value of each m-BCLC model. The original BCLC system showed the largest AIC value, whereas m-BCLC \#2 demonstrated the smallest AIC value, which indicates that m-BCLC $\# 2$ is the most explanatory and informative model. Fig. 5 shows the comparison of IAUC between each original and m-BCLC models. All m-BCLC models showed significantly better prognostic capability than the original BCLC (IAUC, 0.705; 95\% CI, 0.699 to 0.712 ). Among them, m-BCLC \#2 (IAUC, 0.722; 95\% CI, 0.715 to 0.729 ) demonstrated the highest IAUC, followed by m-BCLC \# 1 (IAUC, $0.721 ; 95 \%$ CI, 0.714 to 0.728 ) and m-BCLC
B

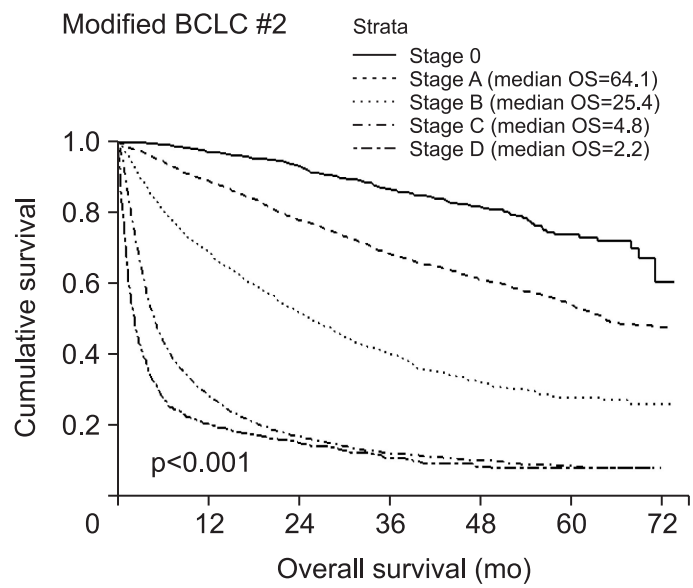

No. of patients at risk

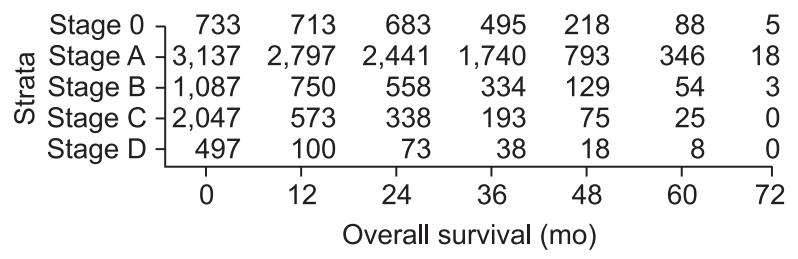

Fig. 4. Comparison of overall survival (OS) according to stage in the modified Barcelona Clinic Liver Cancer (BCLC) models. (A) Comparison of OS according to stage in the modified BCLC \#1. (B) Comparison of OS according to stage in the modified BCLC \#2. (C) Comparison of OS according to stage in the modified BCLC \#3.

\#3 (IAUC, 0.718; 95\% CI, 0.711 to 0.725). By considering AIC and IAUC, m-BCLC \#2 was supposed to be the best explanatory model with good prognostic accuracy. In the comparison of OS according to PS in m-BCLC \#2 model stage C, the patients with PS 0 showed the best OS, followed by the patients with PS 1 and PS 2 (Fig. 2B).

Although overall discrimination function was improved in $\mathrm{m}$ BCLC\#2, the discrimination function between $\mathrm{C}$ and D stage was worse in modified BCLC\#2 (AIC, 0.581; 95\% CI, 0.576 to 0.586) compared to original BCLC (AIC, $0.545 ; 95 \%$ CI, 0.541 to 0.549 ). 
Table 2. Comparison of Prognostic Ability of the Staging Systems

\begin{tabular}{|c|c|c|c|c|c|}
\hline Variable & HR $(95 \%$ CI) & p-value & Linear trend test & $\begin{array}{c}\text { Homogeneity } \\
\text { (likelihood ratio } x^{2} \text { ) }\end{array}$ & $\begin{array}{c}\text { Akaike information } \\
\text { criterion }\end{array}$ \\
\hline Original BCLC & & $<0.001$ & $1,693.2$ & 1,440 & $70,697.17$ \\
\hline Stage 0 & - & & & & \\
\hline Stage A & 2.35 (1.95-2.83) & & & & \\
\hline Stage B & $5.12(4.21-6.24)$ & & & & \\
\hline Stage C & $10.44(8.69-12.55)$ & & & & \\
\hline Stage D & $18.74(15.32-22.93)$ & & & & \\
\hline Modified BCLC \# 1 & & $<0.001$ & $1,950.1$ & 1,707 & $70,126.74$ \\
\hline Stage 0 & - & & & & \\
\hline Stage A & $2.31(1.94-2.76)$ & & & & \\
\hline Stage B & $5.22(4.34-6.28)$ & & & & \\
\hline Stage C & $13.65(11.45-16.28)$ & & & & \\
\hline Stage D & $19.03(15.69-23.09)$ & & & & \\
\hline Modified BCLC \#2 & & $<0.001$ & $1,956.8$ & 1,720 & $70,088.01$ \\
\hline Stage 0 & - & & & & \\
\hline Stage A & 2.33 (1.95-2.78) & & & & \\
\hline Stage B & $5.32(4.42-6.39)$ & & & & \\
\hline Stage C & $14.13(11.85-16.85)$ & & & & \\
\hline Stage D & $19.10(15.74-23.17)$ & & & & \\
\hline Modified BCLC \#3 & & $<0.001$ & $1,866.2$ & 1,668 & $70,212.00$ \\
\hline Stage 0 & - & & & & \\
\hline Stage A & $2.35(1.95-2.84)$ & & & & \\
\hline Stage B & $4.52(3.73-5.47)$ & & & & \\
\hline Stage C & $13.84(11.50-16.65)$ & & & & \\
\hline Stage D & $19.30(15.78-23.61)$ & & & & \\
\hline
\end{tabular}

HR, hazard ratio; CI, confidence interval; BCLC, Barcelona Clinic Liver Cancer.

\section{Proposal for subclassification of $\mathrm{m}-\mathrm{BCLC} \# 2$ stage $\mathrm{C}$}

Although the heterogeneity of stage $\mathrm{C}$ was much improved in $\mathrm{m}$-BCLC\#2, the first treatment option applied and survival were still heterogeneous (Table 3). Therefore, we tried to propose subclassification of m-BCLC\#2 stage C. Cox regression analyses were performed to identify independent risk factors associated with OS of the patients with m-BCLC \#2 stage C (Table 4). Higher CP score (8 or 9: HR, 2.17; 95\% CI, 1.87 to 2.51; $\mathrm{p}<0.001$ ), poorer PS (2: HR, 1.50; 95\% CI, 1.20 to 1.86 ; $\mathrm{p}<0.001$ ), beyond UT7 criteria (HR, 2.56; 95\% CI, 2.19 to 2.99 ; $\mathrm{p}<0.001$ ), and presence of EHS (HR, 1.52; 95\% CI, 1.36 to 1.71 ; $<<0.001$ ) were identified as independent risk factors of poor OS in multivariate Cox regression analysis. By using these variables, which were identified as risk factors for poor prognosis, m-BCLC \#2 C stage was further divided into four groups (C1-4) by considering clinical variables that affect treatment decision (Fig. 6). Patients with a CP score of 8 or 9 or with PS 2 were initially classified into C4. Among the subjects with a CP score of 5, 6, or 7 and with PS 0 or 1, the patients without EHS and within UT7 cri- terion were categorized into $\mathrm{C} 1$, and patients without EHS and beyond UT7 criterion were categorized into C2. The remaining patients with CP scores of 5, 6, and 7 and PS 0 or 1, and with EHS were categorized into C3. Fig. 6 shows the OS distribution according to subgroups $\mathrm{C} 1, \mathrm{C} 2, \mathrm{C} 3$, and C4. The subgroups showed significantly different $\mathrm{OS}$ distribution from stage $\mathrm{C} 1$ to $\mathrm{C} 4$ (all $\mathrm{p}<0.001$ ). Heterogeneity of applied treatment much improved after subclassification of m-BCLC\#2 stage C (Table 3). About 73\% of stage C1 patients were performed local treatment such as resection or transarterial chemoembolization (TACE). In stage C2, TACE was most frequently applied treatment (48.1\%). The proportion of systemic chemotherapy including sorafenib was applied most frequently in stage C3 patients compared with other subclass. In stage C4, more than $50 \%$ of patients were treated with only conservative care.

\section{DISCUSSION}

According to the definition of the BCLC system, BCLC 0, A, and $\mathrm{B}$ include only the patients with PS 0 , while the patients 


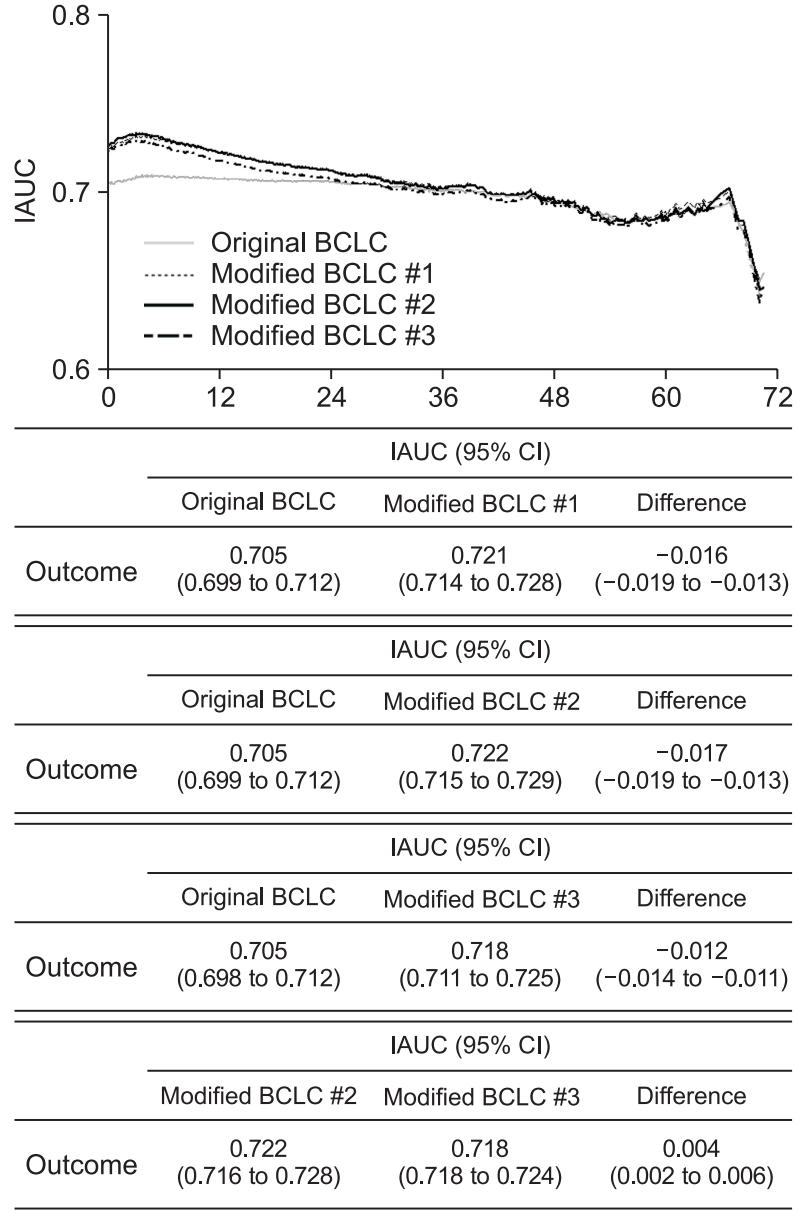

Fig. 5. Comparison of integrated area under the curve (IAUC) between the original and modified Barcelona Clinic Liver Cancer (BCLC) systems.

CI, confidence interval. with PS 1-2 are classified into C stage. Although BCLC system suggests that diminished PS by tumor itself should be considered as the determinant of HCC staging, it is difficult to discriminate whether the deterioration of PS is caused by HCC itself or by other issues including underlying liver disease. Therefore, it causes considerable heterogeneity in the $\mathrm{C}$ stage in the aspect of tumor extent. ${ }^{4}$ The heterogeneity of tumor extent causes discrepancies between treatment guidelines of the BCLC system and real clinical practice as demonstrated in the present study. Therefore, we proposed modification and subclassified BCLC system by reducing the role of PS and giving more weight to tumor extent to derive more clinically relevant staging system.

Hsu et al. ${ }^{15}$ reported that the prognostic accuracy of the BCLC system could be enhanced by modifying the BCLC system based on PS. They proposed three modified BCLC models based on PS, and the best model was included and analyzed as m-BCLC \#3 in the present study. As a result, the modified model proposed by Hsu et al. showed better prognostic accuracy than the original BCLC system. However, m-BCLC \# 1 and m-BCLC \#2 models, which were devised in this study, showed superior predictive performance than the model of Hsu et al. The model with best prognostic capability was identified as m-BCLC \#2, which was devised to put the least emphasis on PS and allocating more weight to tumor extent.

In the $\mathrm{m}$-BCLC \#2 model, stage $\mathrm{C}$ is defined by the presence of preserved liver function (CP class A or B), with PS 0 , 1, or 2, and with VI or EHS. To facilitate treatment decision for patients with stage $C$, we proposed subclassification of stage $C$ in the $m$ BCLC \#2 model by considering clinical and statistical aspect (Fig. 6). C1, C2, and C3 include only patients with relatively preserved liver function (CP scores 5-7) and good PS (PS 0 or 1) that are supposed to be tolerable for specific HCC treatment. ${ }^{16-18}$ C4 includes patients with impaired liver function (CP scores 8-9) and poor PS (PS 2) that are supposed to be not tolerable for spe-

Table 3. Diversity of Applied Treatments for Patients with Modified BCLC \#2 Stage C

\begin{tabular}{|c|c|c|c|c|c|}
\hline & Modified BCLC\#2 stage C & $\mathrm{C} 1$ & $\mathrm{C} 2$ & $\mathrm{C} 3$ & $\mathrm{C} 4$ \\
\hline \multicolumn{6}{|l|}{ First treatment option } \\
\hline Resection & $106(5.2)$ & 27 (17.9) & $27(6.2)$ & $21(4.7)$ & $2(0.4)$ \\
\hline Liver transplantation & $2(0.1)$ & $1(0.7)$ & 0 & 0 & $1(0.2)$ \\
\hline Local ablation therapy & $35(1.7)$ & $7(4.6)$ & $1(0.2)$ & $7(1.5)$ & $8(1.8)$ \\
\hline Transarterial therapy & $793(38.7)$ & $83(55.0)$ & $213(48.1)$ & $175(39.0)$ & $110(24.3)$ \\
\hline Sorafenib & $159(7.8)$ & $2(1.3)$ & $31(7.0)$ & $56(12.5)$ & $37(8.2)$ \\
\hline Arterial infusion chemotherapy & $63(3.1)$ & $4(2.6)$ & $15(3.4)$ & $6(1.3)$ & $12(2.7)$ \\
\hline Systemic therapy other than sorafenib & $136(6.6)$ & $7(4.6)$ & $40(9.0)$ & $37(8.2)$ & $17(3.8)$ \\
\hline Radiation therapy & $70(3.4)$ & $2(1.3)$ & $13(2.9)$ & $16(3.6)$ & $12(2.7)$ \\
\hline Conservative care & $629(30.7)$ & $18(11.9)$ & 95 (21.4) & $119(26.5)$ & 227 (50.2) \\
\hline Missing value & $54(2.6)$ & $0(0)$ & $8(1.8)$ & $12(2.7)$ & 26 (5.7) \\
\hline
\end{tabular}

Data are presented as number (\%).

BCLC, Barcelona Clinic Liver Cancer. 
Table 4. Univariate and Multivariate Analyses of the Variables Associated with Overall Survival in Patients with Modified BCLC \#2 Stage C

\begin{tabular}{|c|c|c|c|c|}
\hline \multirow{2}{*}{ Variable } & \multicolumn{2}{|c|}{ Univariate } & \multicolumn{2}{|c|}{ Multivariate } \\
\hline & HR $(95 \%$ CI) & p-value & HR (95\% CI) & p-value \\
\hline Male sex & $0.96(0.84-1.09)$ & 0.491 & & \\
\hline Age, yr & $1.00(0.99-1.00)$ & 0.382 & & \\
\hline Child-Pugh score, 8 or 9 & $2.03(1.82-2.26)$ & $<0.001$ & $2.17(1.87-2.51)$ & $<0.001$ \\
\hline Platelet, <100 (×109/L) & $1.26(1.19-1.55)$ & $<0.001$ & & \\
\hline Albumin, $\leq 3.5 \mathrm{~g} / \mathrm{L}$ & $1.51(1.38-1.66)$ & $<0.001$ & & \\
\hline Bilirubin, $>2 \mathrm{mg} / \mathrm{dL}$ & $1.80(1.61-2.02)$ & $<0.001$ & & \\
\hline Performance status, 2 & $2.13(1.73-2.62)$ & $<0.001$ & $1.50(1.20-1.86)$ & $<0.001$ \\
\hline Tumor number, $\geq 4$ & $1.74(1.59-1.91)$ & $<0.001$ & & \\
\hline Tumor size, $>5 \mathrm{~cm}$ & $2.17(1.93-2.44)$ & $<0.001$ & & \\
\hline Up-to-7, beyond up-to-7 & $2.35(2.08-2.66)$ & $<0.001$ & $2.56(2.19-2.99)$ & $<0.001$ \\
\hline Vascular invasion, presence & $1.08(0.96-1.22)$ & 0.211 & & \\
\hline Extrahepatic spread, presence & $1.54(1.40-1.69)$ & $<0.001$ & $1.52(1.36-1.71)$ & $<0.001$ \\
\hline
\end{tabular}

BCLC, Barcelona Clinic Liver Cancer; HR, hazard ratio; CI, confidence interval.

cific HCC treatment. C1 and C2 include patients without EHS, but with VI. C1 includes patients with relatively small tumor burden (within UT7) without EHS, whereas C2 includes patients with relatively large tumor burden (beyond UT7) without EHS.

VI has been considered as a contraindication to local therapies, such as TACE and surgical therapy. Therefore, the BCLC system recommends systemic therapy in the form of sorafenib for patients with VI. However, increasing evidence shows that local therapies, including TACE, transarterial radioembolization, radiotherapy, and surgical resection, improve long-term survival in selected patients without major VI. ${ }^{19-28}$ Therefore, the treatment decisions for patients without EHS but only with VI, such as $\mathrm{C} 1$ and $\mathrm{C} 2$ patients, should be individualized considering tumor number, tumor size, tumor location, and degree of VI. C3 includes patients with EHS, preserved liver function, and relatively good PS. Patients with C3 could be treated in the form of systemic therapy, such as molecular targeted therapy including sorafenib.

In the subclassification, we selected UT7 criterion to divide C1 and C2. The UT7 criteria were firstly introduced by the Milan group to select appropriate liver transplantation recipients for expanding the Milan criterion. ${ }^{29}$ The clinical usefulness of this criterion has been validated by several studies. ${ }^{30,31}$ Bolondi et al. ${ }^{5}$ applied the UT7 criterion in the subclassification of patients in BCLC B stage. ${ }^{8}$ They applied this criterion to distinguish major from minor tumor burden by panel discussion of experts, and the predictive capability of the subclassification has been validated..$^{32}$ In the present study, we also selected UT7 criterion as a cutoff value to divide tumor burden into major and minor by considering previous literatures and statistical results. With regard to the underlying liver function, the cutoff value was decided by considering both statistical analysis and previous literature about HCC treatment in CP class B. The treatment for HCC patients with CP class B has been controversial. Recent articles have indicated that patients with a $\mathrm{CP}$ score of 7 have a survival benefit in the treatment of TACE or sorafenib, ${ }^{16-18}$ whereas the survival benefit of patients with a CP score of 8 or 9 was unclear. Survival analysis of our data also demonstrated better prognostic capability when the cutoff value was determined as CP score of 7. Therefore, in this study, patients with a CP score of 5,6 , or 7 were classified as the group with preserved liver function, whereas those with a CP score of 8 or 9 as the group with impaired liver function and categorized into C4 subclass.

This study has several limitations. First, the extent of VI could not be used for subclassification in this study. Several studies have been conducted regarding the subclassification of BCLC C stage. $^{33,34}$ They mainly focused on the extent of VI and the mode of EHS. Both VI and EHS are important variables, and the presence of EHS or VI were identified as independent risk factors for predicting poor OS also in this study. However, the detailed information regarding the extent of VI was lacking in the 2009 to 2013 data of KLCSG, and only the information on the presence of VI is available. Further modification and subclassification considering the extent of VI may be helpful for more detailed and individualized HCC approach. Second, although m-BCLC\#2 showed better overall discrimination function compared to original BCLC, the discrimination function between C and D stage got worse in modified BCLC\#2. It might be caused by reallocation of the PS 1-2 patients without VI or EHS into stage 0, A, or B.

In conclusion, this study developed a more accurate and relevant staging system for patients with HCC by modification and subclassification of the BCLC system, which is expected to improve the heterogeneity of the BCLC C stage. The heterogeneity of the BCLC $\mathrm{C}$ stage hinders the clinical application of the BCLC system by causing discrepancies between the BCLC 
A

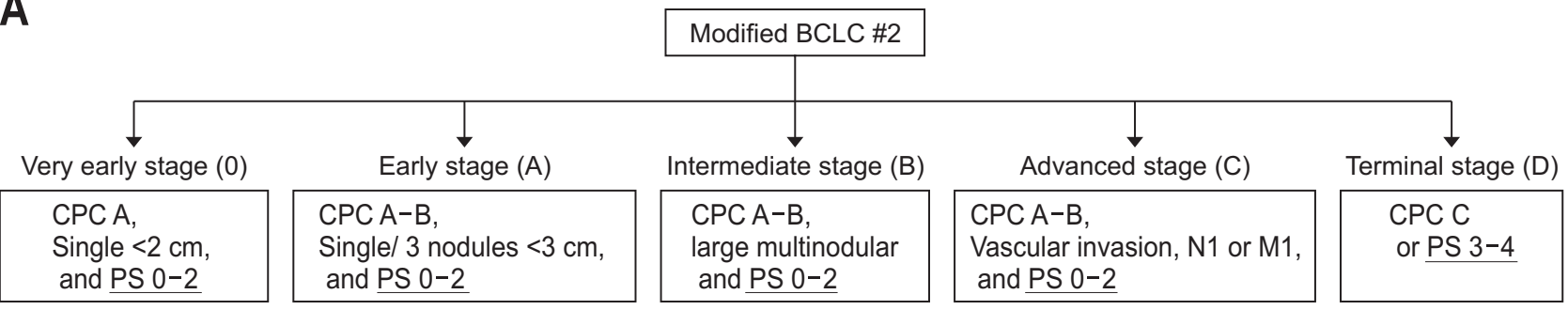

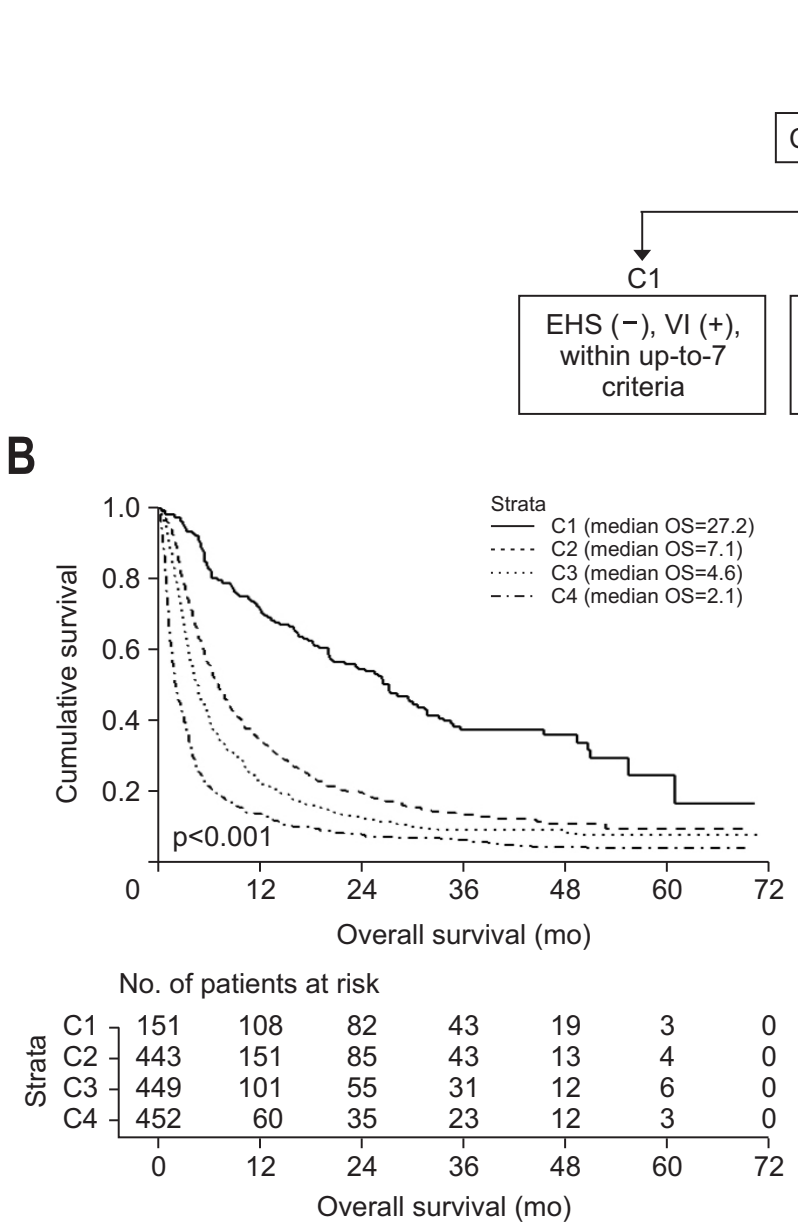

treatment guidelines and clinical practice. This study could help overcome the limitations of the current BCLC system and allow its broader application worldwide. External validation of the proposed modified BCLC model is required to verify its clinical applicability. Careful further modification and subclassification considering real clinical practice could achieve more accurate and applicable staging system for individualized approach in patients with HCC.

\section{CONFLICTS OF INTEREST}

No potential conflict of interest relevant to this article was reported.
Fig. 6. Proposal for subclassification of stage $C$ in the modified Barcelona Clinic Liver Cancer (BCLC) \#2 system. (A) Diagram of the proposed subclassification of stage $\mathrm{C}$ in the modified BCLC \#2 system. (B) Comparison of the overall survival (OS) according to the subclassification of stage $C$ in the modified BCLC \#2 system.

CPC, Child-Pugh class; PS, performance status; CPS, Child-Pugh score; EHS, extrahepatic spread: VI, vascular invasion.

\section{ACKNOWLEDGEMENTS}

This research was supported by the Bio \& Medical Technol ogy Development Program of the National Research Founda tion (NRF) funded by the Korean government (MSIT) (No. NRF2018M3A9E8023861) and a grant of the Korea Health Technology R\&D Project through the Korean Health industry Development Institute (KHIDI), funded by the Ministry of Health \& Welfare, Republic of Korea (grant number: H018C0002).

The authors thank the Korea Central Cancer Registry and Korean Liver Cancer Association. This work received non-financial support from the Research Supporting Program of the Korean Liver Cancer Association based on data from the Primary Liver Cancer Registry, a project supported jointly by the Korean Liver Cancer Association and Korea Central Cancer Registry, Ministry 
of Health and Welfare, Korea.

\section{AUTHOR CONTRIBUTIONS}

Guarantor of the article: J.Y.C. Analysis of data and drafting of the manuscript: H.J.C. Statistical analysis: S.Y.K. Study supervision: M.J.Y., C.K.N., J.C.H., B.M.Y., K.J.L., S.G.L. Study planning: J.H.K., S.J.S., K.M.L. Critical revision of the manuscript for important intellectual content: S.S.K., S.W.C. Study concept: J.Y.C. All authors have approved the final revision.

\section{ORCID}

Jae Youn Cheong https://orcid.org/0000-0001-6246-1783

\section{REFERENCES}

1. Bruix J, Sherman M; American Association for the Study of Liver Diseases. Management of hepatocellular carcinoma: an update. Hepatology 2011;53:1020-1022.

2. Forner A, Llovet JM, Bruix J. Hepatocellular carcinoma. Lancet 2012;379:1245-1255.

3. European Association for the Study of the Liver; European Organisation for Research and Treatment of Cancer. EASL-EORTC clinical practice guidelines: management of hepatocellular carcinoma. J Hepatol 2012;56:908-943.

4. Yu SJ. A concise review of updated guidelines regarding the management of hepatocellular carcinoma around the world: 20102016. Clin Mol Hepatol 2016;22:7-17.

5. Bolondi L, Burroughs A, Dufour JF, et al. Heterogeneity of patients with intermediate (BCLC B) Hepatocellular carcinoma: proposal for a subclassification to facilitate treatment decisions. Semin Liver Dis 2012;32:348-359.

6. Yamakado K, Hirota S. Sub-classification of intermediate-stage (Barcelona Clinic Liver Cancer stage-B) hepatocellular carcinomas. World J Gastroenterol 2015;21:10604-10608.

7. Kudo M, Arizumi T, Ueshima K, Sakurai T, Kitano M, Nishida N. Subclassification of BCLC B stage hepatocellular carcinoma and treatment strategies: proposal of modified Bolondi's subclassification (Kinki criteria). Dig Dis 2015;33:751-758.

8. Ha Y, Shim JH, Kim SO, Kim KM, Lim YS, Lee HC. Clinical appraisal of the recently proposed Barcelona Clinic Liver Cancer stage B subclassification by survival analysis. J Gastroenterol Hepatol 2014;29:787-793.

9. Llovet JM, Brú C, Bruix J. Prognosis of hepatocellular carcinoma: the BCLC staging classification. Semin Liver Dis 1999;19:329-338.

10. Oken MM, Creech RH, Tormey DC, et al. Toxicity and response criteria of the Eastern Cooperative Oncology Group. Am J Clin Oncol 1982;5:649-655.

11. Chen YM, Shih JF, Fan WC, et al. Third-line or fourth-line chemotherapy in non-small-cell lung cancer patients with relatively good performance status. J Chin Med Assoc 2011;74:209-214.
12. Taylor AE, Olver IN, Sivanthan T, Chi M, Purnell C. Observer error in grading performance status in cancer patients. Support Care Cancer 1999;7:332-335.

13. Sørensen JB, Klee M, Palshof T, Hansen HH. Performance status assessment in cancer patients. An inter-observer variability study. Br J Cancer 1993;67:773-775.

14. Chan SC, Fan ST, Chok KS, et al. Survival advantage of primary liver transplantation for hepatocellular carcinoma within the upto-7 criteria with microvascular invasion. Hepatol Int 2011;6:646656.

15. Hsu CY, Lee YH, Hsia CY, et al. Performance status in patients with hepatocellular carcinoma: determinants, prognostic impact, and ability to improve the Barcelona Clinic Liver Cancer system. Hepatology 2013;57:112-119.

16. Hollebecque A, Cattan S, Romano 0, et al. Safety and efficacy of sorafenib in hepatocellular carcinoma: the impact of the ChildPugh score. Aliment Pharmacol Ther 2011;34:1193-1201.

17. Piscaglia F, Terzi E, Cucchetti A, et al. Treatment of hepatocellular carcinoma in Child-Pugh B patients. Dig Liver Dis 2013;45:852858.

18. Hucke F, Sieghart W, Pinter M, et al. The ART-strategy: sequential assessment of the ART score predicts outcome of patients with hepatocellular carcinoma re-treated with TACE. J Hepatol 2014;60:118-126.

19. Chen XP, Qiu FZ, Wu ZD, et al. Effects of location and extension of portal vein tumor thrombus on long-term outcomes of surgical treatment for hepatocellular carcinoma. Ann Surg Oncol 2006;13:940-946.

20. Quirk M, Kim YH, Saab S, Lee EW. Management of hepatocellular carcinoma with portal vein thrombosis. World J Gastroenterol 2015;21:3462-3471.

21. Pinter M, Hucke F, Graziadei I, et al. Advanced-stage hepatocellular carcinoma: transarterial chemoembolization versus sorafenib. Radiology 2012;263:590-599.

22. Luo J, Guo RP, Lai EC, et al. Transarterial chemoembolization for unresectable hepatocellular carcinoma with portal vein tumor thrombosis: a prospective comparative study. Ann Surg Oncol 2011;18:413-420.

23. Kim KM, Kim JH, Park IS, et al. Reappraisal of repeated transarterial chemoembolization in the treatment of hepatocellular carcinoma with portal vein invasion. J Gastroenterol Hepatol 2009;24:806-814

24. Leng JJ, Xu YZ, Dong JH. Efficacy of transarterial chemoembolization for hepatocellular carcinoma with portal vein thrombosis: a meta-analysis. ANZ J Surg 2016;86:816-820.

25. Sangro B, Carpanese L, Cianni R, et al. Survival after yttrium-90 resin microsphere radioembolization of hepatocellular carcinoma across Barcelona Clinic Liver Cancer stages: a European evaluation. Hepatology 2011;54:868-878.

26. Salem R, Lewandowski RJ, Mulcahy MF, et al. Radioembolization for hepatocellular carcinoma using Yttrium-90 microspheres: a comprehensive report of long-term outcomes. Gastroenterology 
2010;138:52-64.

27. Lee JM, Jang BK, Lee YJ, et al. Survival outcomes of hepatic resection compared with transarterial chemoembolization or sorafenib for hepatocellular carcinoma with portal vein tumor thrombosis. Clin Mol Hepatol 2016;22:160-167.

28. Woo HY, Heo J. New perspectives on the management of hepatocellular carcinoma with portal vein thrombosis. Clin Mol Hepatol 2015;21:115-121.

29. Mazzaferro V, Llovet JM, Miceli R, et al. Predicting survival after liver transplantation in patients with hepatocellular carcinoma beyond the Milan criteria: a retrospective, exploratory analysis. Lancet Oncol 2009;10:35-43.

30. D’Amico F, Schwartz M, Vitale A, et al. Predicting recurrence after liver transplantation in patients with hepatocellular carcinoma exceeding the up-to-seven criteria. Liver Transpl 2009;15:12781287.
31. Prasad KR, Young RS, Burra P, et al. Summary of candidate selection and expanded criteria for liver transplantation for hepatocellular carcinoma: a review and consensus statement. Liver Transpl 2011;17:S81-S89.

32. Giannini EG, Moscatelli A, Pellegatta G, et al. Application of the intermediate-stage subclassification to patients with untreated hepatocellular carcinoma. Am J Gastroenterol 2016;111:70-77.

33. Lee S, Kim BK, Song K, et al. Subclassification of Barcelona Clinic Liver Cancer B and C hepatocellular carcinoma: a cohort study of the multicenter registry database. J Gastroenterol Hepatol 2016;31:842-847.

34. Sinn DH, Cho JY, Gwak GY, et al. Different survival of Barcelona Clinic Liver Cancer stage $C$ hepatocellular carcinoma patients by the extent of portal vein invasion and the type of extrahepatic spread. PLoS One 2015;10:e0124434. 